\title{
VERANSTALTUNG
}

\section{Das Lissabon-Urteil des Bundesverfassungsgerichts in der Diskussion}

\author{
Mariella Falkenhain*
}

Am 30. Juni 2009 hat der Zweite Senat des Bundesverfassungsgerichts (BVerfG) sein Urteil zum Vertrag von Lissabon ${ }^{1}$ verkündet. ${ }^{2}$ Die Karlsruher Richter haben entschieden, dass das Zustimmungsgesetz zum Vertrag von Lissabon und die damit verbundene Verfassungsänderung im Kontext der neuen Subsidiaritätsklage des Bundestages mit dem Grundgesetz vereinbar sind. Das Begleitgesetz, mit dem die neuen Rechte des Bundestages und des Bundesrates nach dem Lissabonner Vertrag eingerichtet werden sollten, verstößt aber gegen Art. 38 Abs. 1 in Verbindung mit Art. 23 Abs. 1 Grundgesetz (GG). Die Mitwirkungsrechte des Bundestages und des Bundesrates in europäischen Rechtsetzungs- und Vertragsveränderungsverfahren seien nicht in dem verfassungsrechtlich gebotenen Maße ausgestaltet. Erst nach Inkrafttreten des neugefassten Begleitgesetzes kann die Ratifikationsurkunde hinterlegt werden.

Zwei Wochen nach der Urteilsverkündung diskutierte der Verfassungs- und Europarechtler Prof. Dr. Franz Mayer, Prozessbevollmächtigter des Bundestages im Lissabon-Verfahren, das Urteil mit den Teilnehmern eines Mittagsgespräches des Instituts für Europäische Politik in Berlin.

\section{Ein Urteil, acht Blickwinkel}

Als Ausgangspunkt wählte Mayer einen Klassiker des japanischen Films, um die Viel-

\section{Das Urteil des Bundesverfassungs- gerichts zum Vertrag von Lissabon}

Mittagsgespräch des Instituts für Europäische Politik mit Prof. Dr. Franz C. MAYER LL.M., Universität Bielefeld, mit Unterstützung der Europäischen Kommission sowie der Otto Wolff-Stiftung

Berlin, 15. Juli 2009

Einführung und Moderation: Prof. Dr. Mathias JOPP, Institut für Europäische Politik, Berlin

schichtigkeit und Komplexität des Urteils zu veranschaulichen.

In dem Film „Rashomon“ von Akira Kurosawa (1950) beobachten verschiedene Personen ein Verbrechen, jeder von ihnen schildert das Gesehene dann jedoch völlig anders. Mayer wandelte dieses Szenario auf das Lissabon-Urteil des Bundesverfassungsgerichts $\mathrm{ab}$ und skizzierte acht fiktive Personen (Justus Lipsius, Optimistica, Brutus, Brutalus, Democraticus, Paulus, Nationalus und Publius), die am Abend des 30. Juni 2009 das Urteil aus ihrer Sicht beschreiben und bewerten und dabei jeweils mehr oder weniger akkurat tatsächliche oder vermeintliche Gehalte, Folgen und Deutungsmöglichkeiten des Urteils erfassen.

Eine erste Figur, Justus Lipsius, bezeichnet das Urteil des Bundesverfassungsgerichts als introvertiert, rückwärtsgewandt, feindselig

\footnotetext{
* Mariella Falkenhain, Wissenschaftliche Mitarbeiterin, Institut für Europäische Politik, Berlin.

1 Die gemäß dem Vertrag von Lissabon konsolidierten Fassungen des Vertrags über die Europäische Union (EUV) und des Vertrags über die Arbeitsweise der Europäischen Union (AEUV) sind abgedruckt in: Amtsblatt der EU, Nr. C 115 vom 9. Mai 2008.

2 BverfG, 2 BvE 2/08 vom 30.06.2009.
} 
und in gewissem Sinne sehr deutsch. Seine Kritik umfasst unter anderem die Ausweitung der Zulässigkeit von Verfassungsbeschwerden gegen Europaverträge. Die schon im Maastricht-Urteil ${ }^{3}$ abwegige Konstruktion eines Grundrechts auf einen Abgeordneten, der noch etwas zu sagen hat aus Art. 38 GG, also die ,Verfassungsbeschwerde auf Demokratie', werde noch weiter geöffnet. Da nun auch mit dem Hinweis auf andere Staatsstrukturprinzipien wie Staatlichkeit oder das Sozialstaatsprinzip Verfassungsbeschwerde unter Rückgriff auf Art. 38 GG erhoben werden könne, ${ }^{4}$ solange hinreichender Bezug zur Demokratie hergestellt sei, drohe absehbar eine Klageflut. Um irgendeinen Rechtsschutz gehe es dabei nur in zweiter Linie, vor allem gehe es dem Bundesverfassungsgericht um seine eigenen Zugriffsmöglichkeiten auf den Fortgang der europäischen Integration.

Das binäre Denken des Bundesverfassungsgerichts, das ohne jede Skalierung in der ausschließlichen Gegenüberstellung Staat oder Nicht-Staat, Volk (im Mitgliedstaat) oder Nicht-Volk (auf europäischer Ebene) verharre, führe dazu, dass Demokratie nur in einem europäischen Bundesstaat gedacht werden könne. Der sei jedoch nur über Art. 146 GG, eine neue deutsche Verfassung, erreichbar, also praktisch unmöglich. Aus der dichotomisierenden Logik und der Fixierung auf Volk im Mitgliedstaat folge die Abwertung des Europäischen Parlaments, unter völliger Ausblendung seiner Entwicklung in den letzten 30 Jahren. Der Unionsbürger als Legitimationssubjekt werde komplett ignoriert. Dass dann sogar eine echte Wahl der Europäischen Kommission durch das Europäische Parlament womöglich gegen das Demokratiegebot des Grundgesetzes verstoßen würde, erscheint Lipsius besonders absurd. Dabei sei das Europäische Parlament doch am ehesten in der Lage, die Kommission und intergouvernementale Interessenskartelle der nationa- len Fachminister zu kontrollieren. Die ständige Betonung der souveränen Staatlichkeit erscheine als Abkehr von der bisherigen offenen (völkerrechtsfreundlichen) Staatlichkeit der Bundesrepublik der Gründungsjahre. Es sei ein ,Wir sind wieder wer" in der Sprache des Verfassungsrechts.

Auch die eigene Rollenzuschreibung des Bundesverfassungsgerichts kritisiert Lipsius scharf. Anders als im Maastricht-Urteil sei von einem Kooperationsverhältnis des Bundesverfassungsgerichts zum Europäischen Gerichtshof $(\mathrm{EuGH})$ gar keine Rede mehr. Ganz im Gegenteil laute die Kernaussage aus Karlsruhe: bis hierher und nicht weiter. Die Beanspruchung von einseitigen Letztentscheidungsrechten über Ultra vires-Akte durch das Bundesverfassungsgericht aus dem MaastrichtUrteil werde noch ausgeweitet ${ }^{5}$ und durch die Kontrolle von Übergriffen in die nationale Verfassungsidentität ergänzt. Eine Blockade der europäischen Rechtsentwicklung mit der absehbaren Folge der Flucht ins Informelle und ins Intergouvernementale sei unausweichlich. Die Selbstüberhöhung des Gerichts als Oberaufpasser über die europäische Integration stehe dabei in deutlichem Widerspruch zur Betonung der Bedeutung des Parlaments. Sollten andere Gerichte in der Europäischen Union dem Urteil nacheifern, dann sei das der Spaltpilz für die europäische Integration. Für Lipsius steht fest: Der Bundestag sollte dem Gouvernement des juges endlich Einhalt gebieten.

Die zweite Figur, Optimistica, bezeichnet das Lissabon-Urteil als ein gutes Urteil für Europa, da der Bundestag eine essenzielle Stärkung gegenüber der Bundesregierung erfahre. Der Bundestag habe nun sogar Weisungsrechte gegenüber der Bundesregierung und die Bundesregierung sei etwa in WTO-Fragen zur adäquaten Information des Bundestages verfassungsrechtlich verpflichtet. ${ }^{6}$ Das Urteil

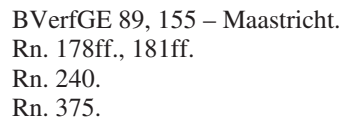


gehe über das Maastricht-Urteil hinaus und mache einiges besser. So werde die Zielvorstellung eines europäischen Bundesstaates erstmals im Grundsatz anerkannt. Die Mitwirkung an der europäischen Integration werde als Verfassungspflicht ${ }^{7}$ beschrieben und der Grundsatz der Europarechtsfreundlichkeit ${ }^{8}$ des Grundgesetzes finde Eingang in die Rechtsprechung des Bundesverfassungsgerichts. Es sei sogar die Rede von einer funktionalen Verfassung auf europäischer Ebene; 9 die Staatsfixiertheit aus dem Maastricht-Urteil sei verschwunden. Deswegen zitierten die Verfassungsrichter Carlo Schmid, ${ }^{10}$ und nicht Carl Schmitt. Optimistica begrüßt, dass der Anwendungsvorrang des Europarechts mehrfach bestätigt wird, und genau besehen dann jetzt offenbar auch der Vorrang des Europarechts über das Verfassungsrecht gelte, bis zur Grenze des Art. 79 Abs. 3 GG. ${ }^{11}$ Zudem werde endlich klargestellt, dass etwaige Reservezuständigkeiten (Ultra vires-Klagen gegen ausbrechende Rechtsakte) auf nationaler Ebene nur dem Bundesverfassungsgericht zukommen, dass diese Reservekontrolle stark zurückgenommen sein müsse und nur ausnahmsweise in offenkundigen Fällen in Betracht kommen könne. ${ }^{12}$ Kurzum: Aus europäischer Sicht werde mit diesem Urteil vieles besser.

Auch Brutus ist mit dem Urteil sehr zufrieden. Seine Begründung zielt jedoch in eine ganz andere Richtung. Er begrüßt die Entscheidung aus Karlsruhe, weil sie das Ende des Vertrages von Lissabon bedeutet. Entscheidend sind für ihn das Junktim zwischen der Neufassung des Begleitgesetzes und der Hinterlegung der Ratifikationsurkunde sowie der Zeitfaktor. Auf die Verzögerung der deutschen Ratifikation möglichst bis ins Jahr 2010 käme es an. Dies stelle sicher, dass der Vertrag von Lissabon nie in Kraft treten werde.
Denn die Verhandlungen über Reichweite und Inhalt des neuen Begleitgesetzes seien absehbar kompliziert und langwierig. Sicherlich werde auch das neue Gesetz wieder vor dem Bundesverfassungsgericht landen, das werde alles viel Zeit brauchen. Somit werde Deutschland den Vertrag von Lissabon vor 2010 nicht ratifizieren können. Entscheidend sei, dass bis dahin die absehbar neu installierte konservative Regierung in Großbritannien wie angekündigt die britische Ratifikation des Vertrages zurückzunehmen werde. Auch wenn das völkerrechtlich gar nicht möglich sei, so sei doch der politische Druck in dem Falle zu groß: Der Vertrag von Lissabon wäre dann beerdigt, ohne je in Kraft getreten zu sein.

Brutalus (Machiavellus) geht es schlicht um die Machtfrage in der Europäischen Union. Er will der Union und insbesondere dem Europäischen Gerichtshof Grenzen aufzeigen und die Macht der souveränen Nationalstaaten in Europa sicherstellen. Deutschland als souveräner Staat und als größter Mitgliedstaat dürfe sich an der europäischen Integration nur insoweit beteiligen, wie es in seinem Interesse liege. Das Konzept der Kontrolle ausbrechender europäischer Rechtsakte, das im Maastricht-Urteil entwickelt wurde, sei ja leider bisher nie aktiviert worden. Mit dem Lissabon-Urteil werde die Kontrolle europäischen Handelns, vor allem des EuGH, konkret. Die Kontrollmöglichkeiten des Bundesverfassungsgerichts über europäisches Handeln würden sogar auf eine Identitätskontrolle ausgeweitet. Für Brutalus wäre dazu ein eigenes, in Art. 93 GG verankertes Verfahren wünschenswert. Mit dem EuGH als Motor der Integration sei dann endlich Schluss.

Alle Staatsgewalt geht vom Volke aus: Democraticus, die nächste Figur, hat ein sehr klassi-
7 Rn. 225.

8 Rn. 225.

9 Rn. 231.

10 Rn. 231.

11 Rn. 240, 332.

12 Rn. 340. 
sches Demokratieverständnis. Für ihn ist nur über das Volk im Mitgliedstaat Demokratie in der Europäischen Union überhaupt vorstellbar. Daher ist er der Ansicht, dass das Europäische Parlament aufgrund seiner nicht gleichheitsgerechten Zusammensetzung (nationale Kontingente) Demokratie nicht vermitteln könne. ${ }^{13}$ Auch der Unionsbürger sei kein taugliches Zurechnungssubjekt für Demokratie auf europäischer Ebene. ${ }^{14}$ Allein der Bundestag, unmittelbar nach den Grundsätzen der freien und gleichen Wahl zusammengesetzt, könne aus der Sicht der deutschen Verfassungsordnung das europäische Konstrukt demokratisch legitimieren. Und Souveränität besteht für ihn vor allem darin, sich notfalls auch von rechtlichen Bindungen lösen $\mathrm{zu}$ können.

Democraticus erhofft sich von der nach dem Urteil eingetretenen Konstellation des Sommers 2009 viel. Die Bedingungen seien nahezu optimal: Die Abgeordneten handelten unter den Bedingungen der Unsicherheit, weil sie nicht wissen könnten, ob sie nach der Wahl zur Opposition oder zur Regierung zählen. Zudem handelten sie, wegen der nahenden Bundestagswahl und der britischen Drohung der Ratifikationsrücknahme, unter Zeitdruck. Und sie würden diesmal keine Anleitung von der Exekutive entgegennehmen. Der Bundestag habe jetzt die einmalige Chance, seine Rechte insbesondere gegenüber der Bundesregierung wirksam festzuschreiben, auch wenn das Urteil zugegebenermaßen allen deutschen Verfassungsorganen eine dauerhafte Integrationsverantwortung zuschreibe. $^{15}$

Paulus sieht im Lissabon-Urteil eine bruchlose Fortsetzung des Maastricht-Urteils. Das Konzept des Staatenverbundes beschreibt für ihn genau das Eigengeartete des Europäischen, und er verweist auf die entsprechende
Passage im Urteil. ${ }^{16}$ Es gehe letztlich eben doch um den Staat.

Nationalus geht es dagegen vor allem um die Wahrung des Nationalen. Das Europäische ist für ihn politischer Sekundärraum, womöglich ohnehin nicht von Dauer. Der Schutz der nationalen Identität, den das Urteil hervorhebt und die Möglichkeit einer Identitätskontrolle durch das Bundesverfassungsgericht begrüßt er. Endlich würden mit dem Urteil konkrete Sachbereiche festgelegt, die auf der nationalen Ebene verbleiben müssen, da sie eines politischen Diskurses mit einer entsprechenden öffentlichen Meinung bedürften. Neben der Staatsbürgerschaft und dem zivilen und militärischen Gewaltmonopol seien das fiskalische Grundentscheidungen zu Einnahmen und Ausgaben einschließlich der Kreditaufnahme, das Strafrecht, Kultur- und Bildungspolitik, die Ordnung der Meinungs-, Presseund Versammlungsfreiheit, der Umgang mit dem religiösen oder weltanschaulichen Bekenntnis sowie sozialstaatliche Grundentscheidungen. ${ }^{17}$ Damit seien der weiteren europäischen Integration klare Grenzen gezogen.

Publius, schließlich, ist ein wahrer Föderalist. Ihn hat das Verfahren bisher kaum interessiert. Er räumt ein, dass die Stimmengewichtung im Bundesrat (etwa zwischen Bremen und Bayern) Ungleichheiten aufweist, die denen ähneln, die das Bundesverfassungsgericht im Europäischen Parlament (Verhältnis großer zu kleiner Mitgliedstaaten) ausmacht und kritisiert. Er sieht ebenfalls ein, dass es im Urteil eigentlich um das Demokratieprinzip mithin den Bundestag - geht, nicht um das Bundesstaatsprinzip, welches den Bundesrat rechtfertigt. Gleichwohl sieht er die machtpolitische Chance, dass die Länder nun bei der Neufassung des Begleitgesetzes schon länger bestehende Wünsche gegenüber der Bundes-

13 Rn. 271, 276ff., 295.

14 Rn. 347ff.

15 Rn. 245.

16 Rn. 229.

17 Rn. 249, 252, 256-260. 
ebene durchsetzen. Schließlich brauche man die Länder für die Verabschiedung des neuen Begleitgesetzes. Insbesondere solle die Bundesregierung endlich gezwungen werden, in Brüssel Länderpositionen $\mathrm{zu}$ vertreten, und diese nicht nur ,maßgeblich zu berücksichtigen'.

Handlungsvorgaben, praktische Folgen und Bewertungen des Richterspruchs

In der Diskussion erläuterte Mayer zunächst, dass die Zielvorgabe, die deutsche Ratifikationsurkunde bis Oktober zu hinterlegen, nicht vom Bundesverfassungsgericht vorgegeben werde, sondern den europapolitischen Zusammenhängen zuzuschreiben sei. $\mathrm{Zu}$ nennen seien hier das Referendum in Irland, die innenpolitische Lage in Großbritannien und die noch ausstehenden Unterschriften des polnischen und tschechischen Staatspräsidenten unter die jeweiligen Ratifikationsurkunden.

Die Vorgaben des Verfassungsgerichts, welche Bundestag und Bundesrat nun unverzüglich umzusetzen hätten, seien sehr konkret und begrenzt, so Mayer weiter. Sie beträfen im Kern lediglich die Stärkung der Mitwirkungsrechte von Bundestag und Bundesrat in Fragen der Fortentwicklung der europäischen Verträge unterhalb der Schwelle der förmlichen Vertragsänderung. Für bestimmte Konstellationen (sogenannte vereinfachte Vertragsänderung, Passerelle-Klauseln, Vertragsabrundung) schreibe das Urteil ein von Bundestag und Bundesrat zu erlassendes $\mathrm{Zu}$ stimmungsgesetz im Sinne von Art. 23 Abs. 1 Satz 2 GG vor, wie es auch bei der förmlichen Vertragsänderung gefordert sei.

Mayer hob in diesem Zusammenhang hervor, dass unmittelbare praktische Folgen des Urteils vor allem die Anwendung der Vertragsabrundungsklausel des Art. 352 AEUV beträfen, weil die gegenwärtige Vorschrift (Art. 308 EG-Vertrag) durchaus häufig zur Anwendung komme (circa 30 Anwendungsbeispiele 2008) und unumstrittene Dinge wie die Fusionskontrollverordnung oder das
ERASMUS-Programm ermöglicht habe. Die Verfassungsrichter hätten die redaktionelle Neuausrichtung der Vertragsabrundungskompetenz im Vertrag von Lissabon (Handeln in den von den Verträgen festgelegten Politikbereichen zur Erreichung der Ziele der Verträge statt Zielverwirklichung im Rahmen des Gemeinsamen Marktes) zum Anlass genommen, diese Bestimmung neu zu bewerten und ihrer Anwendung höhere Hürden aufzuerlegen. Über die Frage, ob mit Art. 352 AEUV tatsächlich eine Neuausrichtung vorliegt, lasse sich aber sehr wohl streiten, so Mayer: Schon über etliche Jahre habe die Ausrichtung auf den Gemeinsamen Markt in der praktischen Handhabung der Kompetenz gar keine große Rolle gespielt, was dem Bundesverfassungsgericht auch bekannt sei. In Deutschland seien nun hohe Hürden für die Nutzung der Abrundungskompetenz errichtet (volles Ratifikationsverfahren mit einem Art. 23-GG-Gesetz). Das könne selbst bei an sich allgemein konsentierten Vorhaben wegen der zeitlichen Verzögerung und den politischen Risiken von Ratifikationsgesetzen, die womöglich eine Zweidrittelmehrheit im Bundestag und Bundesrat - also Einbeziehung der Opposition erfordern, Probleme bereiten. Die daraus resultierende eingeschränkte Anwendbarkeit des Art. 352 AEUV könnte, laut Mayer, zu einer verstärkten Heranziehung der Binnenmarktkompetenz des Art. 95 EG-Vertrag führen. Das sei misslich, weil allseits ja gerade auf eine größere Trennschärfe der Binnenmarktkompetenz hingearbeitet werde, aber unausweichlich, wenn man nicht sinnvolle Regelungen wie eben die Fusionskontrollverordnung versteinern lassen wolle.

Auf die Frage, ob das Bundesverfassungsgericht denn nun Streitigkeiten über Anwendung und Auslegung der europäischen Verträge künftig in einem gesonderten verfassungsgerichtlichen Verfahren selbst entscheiden dürfe, äußerte sich Mayer kritisch. Ein solches Letztentscheidungsrecht sei nicht mit den vertraglichen Verpflichtungen der Bundesrepublik Deutschland, wie sie mit den europäischen Verträgen eingegangen 
wurden, vereinbar. Danach sei alleine der Europäische Gerichtshof in Luxemburg für die Rechtskontrolle auf europäischer Ebene zuständig. Die förmliche Verankerung einer Letztentscheidungskompetenz des deutschen Verfassungsgerichts (über angeblich europäische Kompetenzen überschreitendes Europarecht) im Grundgesetz oder im Bundesverfassungsgerichtsgesetz könne eine Eskalation eines Konflikts zwischen Karlsruhe und Luxemburg nach sich ziehen. Daran könne niemand ein Interesse haben, da der Status quo einigermaßen stabil sei. Ein Vertragsverletzungsverfahren gegen die Bundesrepublik Deutschland sei bei einer formalen Verankerung eines Letztentscheidungsrechts über Europarecht für das Bundesverfassungsgericht, also einer offenen politisch verantworteten Entscheidung des Bundestages für ein solches Recht, aus Brüsseler Sicht nahezu unvermeidbar. Im Übrigen würde die einseitige Beanspruchung jedweder Letztentscheidungsrechte selbst beziehungsweise gerade durch den größten Mitgliedstaat von den anderen Staaten wahrscheinlich nicht akzeptiert werden. Das habe etwas mit Fairness und gleichen Regeln für alle zu tun.

Auf die Frage nach dem Vergleich zu jüngeren Grundsatzurteilen anderer mitgliedstaatlicher Höchstgerichte zur europäischen Integration machte Mayer in der deutschen Entscheidung einen deutlich negativeren Grundton als etwa beim spanischen Verfassungsgericht oder beim französischen Verfassungsrat aus. Im Vergleich sei das deutsche Urteil sehr viel stärker von Abwehr und Misstrauen geprägt. Die europäische Integration werde in weiten Teilen eher als Bedrohung denn als Chance begriffen, im Sinne eines ,Bis hier hin und nicht weiter'. Dabei sei die schematische Gegenüberstellung Europä- ische Union - Mitgliedstaat schon mit Blick auf die in Brüssel im Ministerrat agierenden nationalen Regierungen verfehlt und ignoriere die Mehrebenenstruktur des europäischen Konstrukts.

Auf die Frage nach dem rechtlichen Gehalt des Konzeptes der Verfassungsidentität und ob es dafür klarere rechtliche Konturen gebe als für das Konzept der Subsidiarität erläuterte Mayer, dass sich aus Art. 79 Abs. 3 GG eine Möglichkeit ergebe, die deutsche Verfassungsidentität - rechtlich - näher zu bestimmen. Nach dieser sogenannten Ewigkeitsklausel sind bestimmte Elemente der Verfassung jeglicher Änderung entzogen, nämlich die Unantastbarkeit der Menschenwürde nach Art. 1 GG sowie die Strukturprinzipien aus Art. 20 GG.

Abschließend kommentierte Mayer die nach dem Urteil einsetzenden Aktivitäten. Erstaunt zeigte er sich insbesondere über Forderungen einiger Ländervertreter, ihre Rechte in europapolitischen Entscheidungen umfassend zu stärken. Die Länder meldeten sich völlig ungefragt und mangels Benachteiligung auch grundlos zu Wort. Das Bundesverfassungsgericht fordere mit seinem Urteil schließlich in erster Linie den Bundestag als demokratisch unmittelbar gewählte Volksvertretung dazu auf, aus Gründen der Wahrung des Demokratieprinzips seine Rechte in der europäischen Integration wahrzunehmen.

Zusammenfassend sprach Mayer sich gegen eine voreilige Überbewertung der negativen Aspekte des Urteils aus. Das Lissabon-Urteil dürfte nicht das letzte Wort im Hinblick auf den Fortgang der europäischen Integration gewesen sein, weder für Europa, noch für Deutschland. 